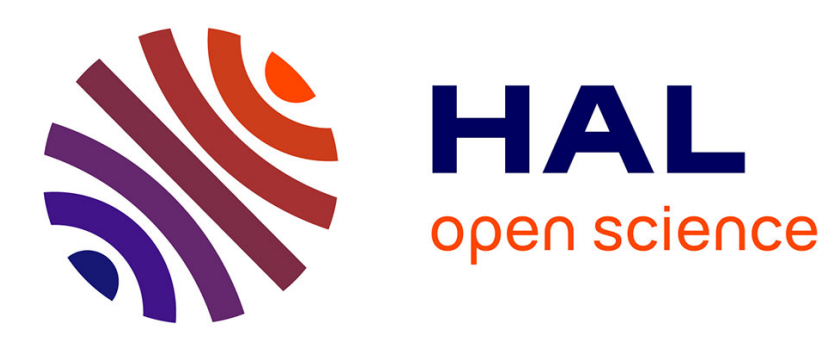

\title{
Finite element discretization of a nonlinear thermoelastic beam model with penalized unilateral contact
}

\author{
Christine Bernardi, Maria Inês M. Copetti
}

\section{To cite this version:}

Christine Bernardi, Maria Inês M. Copetti. Finite element discretization of a nonlinear thermoelastic beam model with penalized unilateral contact. SeMA Journal: Boletin de la Sociedad Española de Matemática Aplicada, 2014, 64 (Issue 1), pp.41-64. 10.1007/s40324-014-0014-9 . hal-00827531

\section{HAL Id: hal-00827531 \\ https://hal.science/hal-00827531}

Submitted on 29 May 2013

HAL is a multi-disciplinary open access archive for the deposit and dissemination of scientific research documents, whether they are published or not. The documents may come from teaching and research institutions in France or abroad, or from public or private research centers.
L'archive ouverte pluridisciplinaire HAL, est destinée au dépôt et à la diffusion de documents scientifiques de niveau recherche, publiés ou non, émanant des établissements d'enseignement et de recherche français ou étrangers, des laboratoires publics ou privés. 


\title{
Finite element discretization of a thermoelastic beam
}

\author{
by Christine Bernardi ${ }^{1}$ and Maria Inês M. Copetti ${ }^{3}$
}

\begin{abstract}
We consider the steady case of a nonlinear model for a thermoelastic beam that can enter in contact with obstacles. We first prove the well-posedness of this problem. Next, we propose a finite element discretization and perform the a priori and a posteriori analysis of the discrete problem. Some numerical experiments confirm the interest of this approach.
\end{abstract}

Résumé: Nous considérons le cas stationnaire d'un modèle non linéaire pour une poutre thermoélastique qui peut entrer en contact avec des obstacles. Nous prouvons que ce problème est bien posé. Puis nous écrivons une discrétisation par éléments finis et effectuons l'analys a priori et a posteriori du problème discret. Quelques expériences numériques confirment l'intérêt de cette approche.

1 Laboratoire Jacques-Louis Lions, C.N.R.S. \& Université Pierre et Marie Curie,

B.C. 187, 4 place Jussieu, 75252 Paris Cedex 05, France.

3 LANA, Departamento de Matemática, Universidade Federal de Santa Maria, 97105-900, Santa Maria, RS Brasil.

e-mail addresses: bernardi@ann.jussieu.fr, Adel.Blouza@univ-rouen.fr,mimcopetti@ufsm.br 



\section{Introduction.}

We consider in this work a homogeneous, thermoviscoelastic thick beam whose reference configuration is the interval $I=] 0,1[$. The beam is rigidly attached at its left end while its right end is free to come into frictionless contact with two pointed reactive obstacles located at the vertical positions $g_{1}$ and $g_{2}$. A nonlinearity of Kirchhoff type taking into account changes in tension due to variations in the displacement is incorporated in the model and the temperature of the beam is prescribed at both ends.

Let $u(x, t), \phi(x, t)$ and $\theta(x, t)$ denote the vertical displacement, the angular rotation of cross section and the temperature along the transversal direction of the beam, respectively. Thus, the motion and thermal evolution of the beam are described by the system of equations

$$
\begin{aligned}
& u_{t t}=u_{x x}-\phi_{x}+\zeta\left(u_{x x t}-\phi_{x t}\right)+\left(\beta+\rho \int_{0}^{1} u_{x}^{2} d x\right) u_{x x}, x \in I, t>0, \\
& \phi_{t t}=\phi_{x x}+u_{x}-\phi+\zeta\left(\phi_{x x t}+u_{x t}-\phi_{t}\right)-a \theta_{x}, x \in I, t>0 \\
& \theta_{t}=\theta_{x x}-a \phi_{x t}, x \in I, t>0
\end{aligned}
$$

with the initial conditions

$$
\begin{aligned}
u(x, 0) & =u_{0}(x), u_{t}(x, 0)=u_{1}(x), x \in I, \\
\phi(x, 0) & =\phi_{0}(x), \phi_{t}(x, 0)=\phi_{1}(x), x \in I, \\
\theta(x, 0) & =\theta_{0}(x), x \in I,
\end{aligned}
$$

and the boundary conditions

$$
\begin{aligned}
& u(0, t)=\phi(0, t)=0, t>0 \\
& \phi_{x}(1, t)+\zeta \phi_{x t}(1, t)=0, t>0 \\
& \theta(0, t)=\theta_{A}, \theta(1, t)=0, t>0 \\
& \sigma(1, t)=-\frac{1}{\varepsilon}\left(\left[u(1, t)-g_{2}\right]_{+}-\left[g_{1}-u(1, t)\right]_{+}\right), t>0 .
\end{aligned}
$$

Here, the stress field is given by $\sigma=u_{x}-\phi+\zeta\left(u_{x t}-\phi_{t}\right)+\left(\beta+\rho \int_{0}^{1} u_{x}^{2} d x\right) u_{x}$, where $\zeta>0$ is a viscosity coefficient, $\beta$ accounts for an axial force at rest and $\rho>0$ is a constant related to the material. Finally, we assume that $g_{1} \leq 0 \leq g_{2}$. The symbol $[f]_{+}$denotes the positive part $\max \{f, 0\}$ of a function $f$.

The boundary condition in the fourth line of (1.3) with $\varepsilon>0$ is called normal compliance condition. In the limit $\varepsilon \rightarrow 0$ the obstacles become rigid and a Signorini condition is obtained. The condition in the first line of (1.3) is assumed at $x=0$ on the two unknowns $u$ and $\phi$, it means that the beam is clamped at this point. We remark that the temperature acts on the motion of $\phi$ directly and indirectly on the displacement $u$ through the coupling 
of the equations. We refer to the works of Lagnese, Leugering and Schmidt [7], Sapir and Reiss [10] and Anh and Stewart [1] for details on the modelling.

In this work, we are only interested in the steady situation. Indeed, stationary problems for nonlinear, elastic, Euler-Bernoulli beams were considered previously in [9], [12], [8]. An algorithm based on the Galerkin method was proposed by Peradze [9] to solve an equation for a simply supported beam, while the influence of an external load was investigated by Zelati, Giorgi and Pata [12]. In [8], Ma included a nonlinear boundary condition, established existence of solutions and presented a numerical algorithm using the finite difference method. A dynamic, nonlinear, elastic, Timoshenko beam problem was studied by Sapir and Reiss [10]. In particular, stationary solutions were described and their stability investigated. Recently, the quasi-static thermoviscoelastic nonlinear contact problem for an Euler-Bernoulli beam was numerically studied by Copetti and Fernández [4]; in the latter case, a finite element discretization was proposed and analyzed and some numerical experiments were performed. However, to our knowledge, the present paper is the first work to consider a nonlinear, thermoelastic, Timoshenko beam model with contact boundary conditions.

It can be noted that problem (1.1) when suppressing all time derivatives, results into a system of ordinary differential equations. However in view of the discretization we write its variational formulation. Even if the problem is simpler, proving its well-posedness is not at all obvious due to the nonlinear terms. We study first the linear problem, next we establish the well-posedness of the nonlinear problem under some conditions on the coefficients.

Next, we propose a finite element discretization of this problem. We then perform its a priori and a posteriori analysis. For both of them, the approach is the same: first studying the linear problem, next extending by hand the results to the nonlinear problem. All this justifies the choice of the discretization that we have made. Finally, we present and analyze an iterative algorithm for solving the nonlinear discrete problem. Some numerical experiments confirm the interest of our approach.

The outline of this article is as follows.

- In Section 2, we present the steady problem and its variational formulation. We prove its well-posedness.

- Section 3 is devoted to the description and a priori analysis of the discrete problem.

- The a posteriori analysis of the discretization is performed in Section 4.

- An iterative algorithm for solving the discrete problem is studied in Section 5.

- In Section 6, we present a few numerical experiments.

Acknowledgement: The work of M.I.M. Copetti was partially supported by the Brazilian institution CAPES under grant 8659-11-7. She also wishes to thank the Laboratoire Jacques-Louis Lions of Université Pierre et Marie Curie for the hospitality during the course of this work. 


\section{The continuous problem and its well-posedness.}

When forgetting all dependency with respect to $t$ of the unknowns, we observe that the temperature $\theta$ satisfies $\theta_{x x}=0$, so that in view of the boundary conditions in the third line of (1.3) it is given by

$$
\theta(x)=\theta_{A}(1-x)
$$

So we are led to consider the system with two unknowns, where $I$ stands for the interval ] $0,1[$,

$$
\begin{cases}-u_{x x}+\phi_{x}-N(u) u_{x x}=0 & \text { in } I, \\ -\phi_{x x}-u_{x}+\phi=a \theta_{A} & \text { in } I \\ u(0)=\phi(0)=0, & \\ \phi_{x}(1)=0, \quad \sigma(u, \phi)(1)=g(u)(1), & \end{cases}
$$

where the quantity $\sigma(u, \phi)$ is defined by

$$
\sigma(u, \phi)=u_{x}-\phi+N(u) u_{x}
$$

The nonlinear term $N(u)$ reads

$$
N(u)=\beta+\rho|u|_{H^{1}(I)}^{2},
$$

where the constant $\rho$ is positive. We also recall that the boundary quantity $g(u)$ is equal to, with $g_{1} \leq 0 \leq g_{2}$,

$$
g(u)(1)= \begin{cases}-\frac{1}{\varepsilon}\left(u(1)-g_{2}\right) & \text { if } u(1) \geq g_{2}, \\ 0 & \text { if } g_{1} \leq u(1) \leq g_{2} \\ -\frac{1}{\varepsilon}\left(u(1)-g_{1}\right) & \text { if } u(1) \leq g_{1}\end{cases}
$$

Let $H_{*}^{1}(I)$ stand for the space of functions in $H^{1}(I)$ which cancel at 0 . We now consider the variational problem

Find $(u, \phi)$ in $H_{*}^{1}(I)^{2}$ such that

$$
\begin{array}{ll}
\forall w \in H_{*}^{1}(I), \quad\left(u_{x}, w_{x}\right)-\left(\phi, w_{x}\right)+\left(N(u) u_{x}, w_{x}\right)=g(u)(1) w(1), \\
\forall \chi \in H_{*}^{1}(I), \quad\left(\phi_{x}, \chi_{x}\right)-\left(u_{x}, \chi\right)+(\phi, \chi)=\left(a \theta_{A}, \chi\right) .
\end{array}
$$

Indeed, the next lemma is an easy consequence of the density of $\mathcal{D}(10,1])$ in $H_{*}^{1}(I)$.

Lemma 2.1. Problem (2.2) is equivalent to system (2.1) (when satisfied in the distribution sense).

\subsection{The linear problem.}


In a first step, we assume that $N(u)$ is zero and we take $g(u)(1)$ equal to a constant $g$. We set: $\mathbb{X}=H_{*}^{1}(I)^{2}$ and we equip it with the semi-norm

$$
\|(u, \phi)\|_{\mathbb{X}}=\left(|u|_{H^{1}(I)}^{2}+|\phi|_{H^{1}(I)}^{2}\right)^{\frac{1}{2}} .
$$

Indeed, it follows from the Poincaré-Friedrichs inequality that this quantity is a norm on $\mathbb{X}$, equivalent to $\|\cdot\|_{H^{1}(I)^{2}}$. For all $(u, \phi)$ and $(w, \chi)$ in $\mathbb{X}$, we introduce the bilinear form

$$
a(u, \phi ; w, \chi)=\left(u_{x}, w_{x}\right)-\left(\phi, w_{x}\right)+\left(\phi_{x}, \chi_{x}\right)-\left(u_{x}, \chi\right)+(\phi, \chi),
$$

so that problem (2.2) can equivalently be written

Find $(u, \phi)$ in $\mathbb{X}$ such that

$$
\forall(w, \chi) \in \mathbb{X}, \quad a(u, \phi ; w, \chi)=g w(1)+\left(a \theta_{A}, \chi\right)
$$

The continuity of the form $a(\cdot ; \cdot)$ on $\mathbb{X} \times \mathbb{X}$ is obvious. In the next lemma, we study its ellipticity.

Lemma 2.2. The form $a(\cdot ; \cdot)$ satisfies the following ellipticity property, for a constant $\alpha>0$,

$$
\forall(u, \phi) \in \mathbb{X}, \quad a(u, \phi ; u, \phi) \geq \alpha\|(u, \phi)\|_{\mathbb{X}}^{2}
$$

Proof: We have

$$
a(u, \phi ; u, \phi)=\left\|u_{x}-\phi\right\|_{L^{2}(I)}^{2}+|\phi|_{H^{1}(I)}^{2} .
$$

Since

$$
\left\|u_{x}-\phi\right\|_{L^{2}(I)}^{2}=\left\|u_{x}\right\|_{L^{2}(I)}^{2}-2\left(u_{x}, \phi\right)+\|\phi\|_{L^{2}(I)}^{2},
$$

the Young's inequality yields, for $0<\eta<1$,

$$
\left\|u_{x}-\phi\right\|_{L^{2}(I)}^{2} \geq(1-\eta)\left\|u_{x}\right\|_{L^{2}(I)}^{2}+\frac{\eta-1}{\eta}\|\phi\|_{L^{2}(I)}^{2} .
$$

Owing to the Poincaré-Friedrichs inequality, we have $\|\phi\|_{L^{2}(I)} \leq \frac{1}{\sqrt{2}}|\phi|_{H^{1}(I)}$, whence

$$
a(u, \phi ; u, \phi) \geq(1-\eta)|u|_{H^{1}(I)}^{2}+\left(1-\frac{1}{2} \frac{1-\eta}{\eta}\right)|\phi|_{H^{1}(I)}^{2}
$$

An appropriate choice of $\eta$ (more precisely, taking $\eta=\frac{1}{2}$ ) gives the desired result with $\alpha=\frac{1}{2}$.

The well-posedness of problem (2.3) is a direct consequence of this lemma, combined with the Lax-Milgram lemma.

Proposition 2.3. For any constants $g$, $a$ and $\theta_{A}$, problem (2.3) admits a unique solution. 


\subsection{The full problem.}

We go back to problem (2.2) and first prove a boundedness propety for its solution.

Lemma 2.4. For all $\beta>-\alpha$, the part $u$ of any solution $(u, \phi)$ of problem (2.2) satisfies

$$
|u|_{H^{1}(I)} \leq c\left|a \theta_{A}\right|
$$

where the constant $c$ only depends on $\alpha$ and $\alpha+\beta$.

Proof: Combining the ellipticity property of the form $a(\cdot, \cdot)$, see Lemma 2.2, with the definition of the nonlinear term $N(u)$ gives

$$
(\alpha+\beta)|u|_{H^{1}(I)}^{2}+\alpha|\phi|_{H^{1}(I)}^{2}-g(u)(1) u(1) \leq\left|a \theta_{A}\right|\|\phi\|_{L^{2}(I)} .
$$

It is readily checked that, in all cases, the quantity $-g(u)(1) u(1)$ is nonnegative. Thus the desired result follows from the Poincaré-Friedrichs inequality.

Let us now introduce the mapping $\Phi$ from $\mathbb{X}$ into itself by

$$
\langle\Phi(u, \phi),(w, \chi)\rangle=a(u, \phi ; w, \chi)+\left(N_{z}(u) u_{x}, w_{x}\right)-g(u)(1) w(1)-\left(a \theta_{A}, \chi\right),
$$

where the quantities $N_{z}(u)$ and $N_{z}^{*}(u)$ are defined, for a function $z$ in $H_{*}^{1}(I)$, by

$$
N_{z}(u)=\beta+\rho\left|\left(z_{x}, u_{x}\right)\right|, \quad N_{z}^{*}(u)=\rho\left|\left(z_{x}, u_{x}\right)\right| .
$$

In a first step, we wish to prove that the problem

$$
\forall(w, \chi) \in \mathbb{X}, \quad\langle\Phi(u, \phi),(w, \chi)\rangle=0,
$$

has a solution. The key idea for this consists in applying Brouwer's fixed point theorem.

Proposition 2.5. There exists a real number $\beta_{0}>-1$ such that, for all $\beta>\beta_{0}$ and all data $g_{1}, g_{2}, a$ and $\theta_{A}$, and also for any function $z$ in $H_{*}^{1}(I)$, problem (2.5) admits a solution.

Proof: We proceed in three steps.

1) The mapping $\Phi$ is clearly continuous from $\mathbb{X}$ into itself. To go further, we use Lemma 2.2 combined with the imbedding of $H_{*}^{1}(I)$ in $\mathcal{C}^{0}([0,1])$. We also recall that the quantity $-g(u)(1) u(1)$ is nonnegative. All this yields that, for all $(u, \phi)$ satisfying $|u|_{H^{1}(I)}=\mu$ and $|\phi|_{H^{1}(I)}=\mu$,

$$
\langle\Phi(u, \phi),(u, \phi)\rangle \geq(2 \alpha+\beta) \mu^{2}-c \mu,
$$

where $c$ only depends on the product $\left|a \theta_{A}\right|$. So, for $\beta>\beta_{0}>-2 \alpha$, and $\mu$ equal to $\frac{2 c}{2 \alpha+\beta}$, the quantity $\langle\Phi(u, \phi),(u, \phi)\rangle$ is positive on the tensorized sphere of $\mathbb{X}$ with radius $\mu$.

2) Since $H_{*}^{1}(I)$ is separable, there exists an increasing sequence $\left(\mathbb{X}_{n}\right)_{n}$ of finite-dimensional 
subspaces of $\mathbb{X}$ such that their union is dense in $\mathbb{X}$. The properties of the mapping $\Phi$ on $\mathbb{X}_{n}$ being exactly the same as on $\mathbb{X}$, Brouwer's theorem [6, chap. IV, cor. 1.1$]$ implies that there exists a unique $\left(u_{n}, \phi_{n}\right)$ in the tensorized ball of $\mathbb{X}_{n}$ with radius $\mu$ satisfying

$$
\forall(w, \chi) \in \mathbb{X}_{n}, \quad\left\langle\Phi\left(u_{n}, \phi_{n}\right),(w, \chi)\right\rangle=0 .
$$

3) Since the sequence $\left(u_{n}, \phi_{n}\right)_{n}$ is bounded in $\mathbb{X}$, there exists a subsequence, still denoted by $\left(u_{n}, \phi_{n}\right)_{n}$ for simplicity, which converges to a pair $(u, \phi)$ weakly in $\mathbb{X}$. The sequence $\left(\mathbb{X}_{n}\right)_{n}$ being increasing, equation (2.5) can be re-written, for all $m \leq n$,

$$
\forall\left(w_{m}, \chi_{m}\right) \in \mathbb{X}_{m}, \quad\left\langle\Phi\left(u_{n}, \phi_{n}\right),\left(w_{m}, \chi_{m}\right)\right\rangle=0,
$$

We now pass to the limit on $n$ (indeed, the problem only contains linear terms), then on $m$. This yields that $(u, \phi)$ is a solution of problem $(2.5)$.

We are now in a position to prove the main result of this section. However a technical lemma is needed for that.

Lemma 2.6. For all functions $u_{1}$ and $u_{2}$ in $H_{*}^{1}(I)$, the quantity

$$
X\left(u_{1}, u_{2}\right)=-\left(g\left(u_{1}\right)(1)-g\left(u_{2}\right)(1)\right)\left(u_{1}-u_{2}\right)(1)
$$

is nonnegative.

Proof: Owing to the symmetry property $X\left(u_{2}, u_{1}\right)=X\left(u_{1}, u_{2}\right)$, we assume without restriction that: $u_{1}(1) \geq u_{2}(1)$. We now consider the different values of $u_{2}(1)$.

1) If $u_{2}(1) \geq g_{2}$, the quantity $X\left(u_{1}, u_{2}\right)$ satisfies

$$
X\left(u_{1}, u_{2}\right)=\frac{1}{\varepsilon}\left(u_{1}-u_{2}\right)(1)\left(u_{1}-u_{2}\right)(1) \geq 0 .
$$

2) If $g_{1} \leq u_{2}(1) \leq g_{2}$, it is readily checked that $g\left(u_{2}\right)(1)=0$. The quantity $-g\left(u_{1}\right)(1)$ is either equal to $\frac{1}{\varepsilon}\left(u_{1}-g_{2}\right)$ when $u_{1}(1) \geq g_{2}$ or to zero otherwise. In all cases, we have $X\left(u_{1}, u_{2}\right) \geq 0$.

3) If $u_{2}(1) \leq g_{1}$, we observe that

$$
\begin{aligned}
& \frac{X\left(u_{1}, u_{2}\right)}{\left(u_{1}-u_{2}\right)(1)} \\
& =-\frac{1}{\varepsilon}\left(u_{2}(1)-g_{1}\right)+ \begin{cases}\frac{1}{\varepsilon}\left(u_{1}(1)-g_{1}\right)=\frac{1}{\varepsilon}\left(u_{1}-u_{2}\right)(1) & \text { if } u_{1}(1) \leq g_{1}, \\
0=\frac{1}{\varepsilon}\left(g_{1}-u_{2}(1)\right) & \text { if } g_{1} \leq u_{1}(1) \leq g_{2}, \\
\frac{1}{\varepsilon}\left(u_{1}(1)-g_{2}\right)=\frac{1}{\varepsilon}\left(\left(u_{1}-u_{2}\right)(1)+g_{1}-g_{2}\right) & \text { if } u_{1}(1) \geq g_{2} .\end{cases}
\end{aligned}
$$

By noting that $\left(u_{1}-u_{2}\right)(1) \geq g_{2}-g_{1}$ in this last case, we also derive that $X\left(u_{1}, u_{2}\right) \geq 0$.

Theorem 2.7. There exists a real number $\rho_{0}>0$ such that, for all $\rho<\rho_{0}$ and $\beta>\beta_{0}$ and all data $g_{1}, g_{2}, a$ and $\theta_{A}$, problem (2.2) admits at least a solution. 
Proof: Let $\Psi$ denote the mapping from $H_{*}^{1}(I)$ into itself which associates with $z$ the part $u$ of the solution $(u, \phi)$ of problem $(2.5)$ exhibited in Proposition 2.5. For all functions $z_{1}$ and $z_{2}$ in $H_{*}^{1}(I)$, we set: $u_{1}=\Psi\left(z_{1}\right)$ and $u_{2}=\Psi\left(z_{2}\right)$. We see that, for any $(w, \chi)$ in $\mathbb{X}$, and with obvious notation for $\phi_{1}$ and $\phi_{2}$,

$$
\begin{aligned}
a\left(u_{1}-u_{2}, \phi_{1}-\phi_{2} ; w, \chi\right)+\beta\left(u_{1 x}\right. & \left.-u_{2 x}, w_{x}\right)-g\left(u_{1}\right)(1) w(1)+g\left(u_{2}\right)(1) w(1) \\
& =-\left(N_{z_{1}}^{*}\left(u_{1}\right) u_{1 x}-N_{z_{2}}^{*}\left(u_{2}\right) u_{2 x}, w_{x}\right) .
\end{aligned}
$$

We now take $(w, \chi)$ equal to $\left(u_{1}-u_{2}, \phi_{1}-\phi_{2}\right)$. We deduce from Lemmas 2.2 and 2.6

$$
(\alpha+\beta)\left|u_{1}-u_{2}\right|_{H^{1}(I)} \leq\left|N_{z_{1}}^{*}\left(u_{1}\right) u_{1}-N_{z_{2}}^{*}\left(u_{2}\right) u_{2}\right|_{H^{1}(I)} .
$$

If $\mu$ is the real number introduced in the proof of Proposition 2.5, we now assume that $z_{1}$ and $z_{2}$ belong to the ball $\mathcal{B}$ of $\mathbb{X}$ with radius $\mu$, and we observe that $u_{1}$ and $u_{2}$ belong to this same ball; then, several triangle inequalities lead to

$$
(\alpha+\beta)\left|u_{1}-u_{2}\right|_{H^{1}(I)} \leq \rho c \mu^{2}\left(\left|u_{1}-u_{2}\right|_{H^{1}(I)}+\left|z_{1}-z_{2}\right|_{H^{1}(I)}\right) .
$$

Next, we choose $\rho_{0}$ such that $\rho_{0} c \mu^{2}<\frac{\alpha+\beta}{2}$. We thus derive

$$
\left|u_{1}-u_{2}\right|_{H^{1}(I)} \leq C\left|z_{1}-z_{2}\right|_{H^{1}(I)},
$$

for a constant $C<1$. Consequently, the mapping $\Psi$ is a contraction of $\mathcal{B}$ and then admits a unique fixed point $u$ dans $\mathcal{B}$. The pair $(u, \phi)$, where $\phi$ is the solution of the second line of (2.2) for the datum $u_{x}$ (its existence and uniqueness are obvious), is a solution of problem $(2.2)$.

It can be noted that the conditions on $\beta$ and $\rho$ which appear in the statement of Theorem 2.6 seem too strong for the existence result (think of applying a Cauchy-Lipschitz theorem). We conclude this section with the uniqueness result.

Theorem 2.8. For all $\beta>-\alpha$ and all data $g_{1}, g_{2}, a$ and $\theta_{A}$, problem (2.2) admits at most a solution.

Proof: Let $\left(u_{1}, \phi_{1}\right)$ and $\left(u_{2}, \phi_{2}\right)$ be two solutions of problem $(2.2)$. The same arguments as in the previous proof imply that

$$
\begin{gathered}
a\left(u_{1}-u_{2}, \phi_{1}-\phi_{2} ; w, \chi\right)+\beta\left(u_{1 x}-u_{2 x}, w_{x}\right)-g\left(u_{1}\right)(1) w(1)+g\left(u_{2}\right)(1) w(1) \\
+\left(N_{u_{1}}^{*}\left(u_{1}\right) u_{1 x}-N_{u_{2}}^{*}\left(u_{2}\right) u_{2 x}, w_{x}\right)=0 .
\end{gathered}
$$

We now take $(w, \chi)$ equal to $\left(u_{1}-u_{2}, \phi_{1}-\phi_{2}\right)$. Lemma 2.6 implies that the quantity $-\left(g\left(u_{1}\right)(1)-g\left(u_{2}\right)(1)\right)\left(u_{1}-u_{2}\right)(1)$ is nonnegative. On the other hand, we have the inequalities, see [3, Proposition 1] for their proof,

$$
\begin{aligned}
& N_{u_{1}}^{*}\left(u_{1}\right)\left(u_{1 x}, u_{1 x}-u_{2 x}\right) \geq \frac{1}{4}\left|u_{1}\right|_{H^{1}(I)}^{4}-\frac{1}{4}\left|u_{2}\right|_{H^{1}(I)}^{4}, \\
& \quad-N_{u_{2}}^{*}\left(u_{2}\right)\left(u_{2 x}, u_{1 x}-u_{2 x}\right) \geq \frac{1}{4}\left|u_{2}\right|_{H^{1}(I)}^{4}-\frac{1}{4}\left|u_{1}\right|_{H^{1}(I)}^{4} .
\end{aligned}
$$


Combining all this with Lemma 2.2 yields

$$
(\alpha+\beta)\left|u_{1}-u_{2}\right|_{H^{1}(I)}^{2} \leq 0,
$$

so that $u_{1}$ and $u_{2}$ coincide. The functions $\phi_{1}$ and $\phi_{2}$ then, satisfy

$$
\forall \chi \in H_{*}^{1}(I), \quad\left(\phi_{1 x}-\phi_{2 x}, \chi_{x}\right)+\left(\phi_{1}-\phi_{2}, \chi\right)=0 .
$$

Taking $\chi$ equal to $\phi_{1}-\phi_{2}$ yields that they also coincide. This proves the uniqueness of the solution of problem (2.2). 


\section{The finite element discrete problem and its a priori analysis.}

We introduce a regular family $\left(\mathcal{T}_{h}\right)_{h}$ of triangulations of $I$ by closed intervals in the sense that, for each $h$,

- The closure $\bar{I}=[0,1]$ of $I$ is the union of all elements of $\mathcal{T}_{h}$;

- The intersection of two different elements of $\mathcal{T}_{h}$ is either empty or an endpoint of both of them;

- If two intervals $K$ and $K^{\prime}$ of $\mathcal{T}_{h}$ are adjacent, i.e. share an endpoint, their lengths $h_{K}$ and $h_{K^{\prime}}$ satisfy

$$
h_{K} \leq \sigma h_{K^{\prime}}
$$

for a constant $\sigma$ independent of $h$.

As usual, $h$ stands for the maximum of the lengths $h_{K}, K \in \mathcal{T}_{h}$. In what follows, $c, c^{\prime}, \ldots$ stand for generic constants that may vary from line to line but are always independent of the parameter $h$.

For a positive integer $k$ and each $K$ in $\mathcal{T}_{h}$, we introduce the space $\mathcal{P}_{k}(K)$ of restrictions to $K$ of polynomials with one variable and degree $\leq k$. Then, we define the discrete spaces

$$
\mathbb{Y}_{h}=\left\{\mu_{h} \in H_{*}^{1}(I) ; \forall K \in \mathcal{T}_{h},\left.\mu_{h}\right|_{K} \in \mathcal{P}_{k}(K)\right\}, \quad \mathbb{X}_{h}=\mathbb{Y}_{h}^{2}
$$

Due to the imbedding of $\mathbb{Y}_{h}$ into $H_{*}^{1}(I)$, the discretization that we consider is fully conforming.

\subsection{The linear problem.}

As previously, we assume that $N(u)$ is zero and we take $g(u)(1)$ equal to a constant $g$. The discrete problem is constructed from the variational problem (2.3) by the Galerkin method. So, it reads

Find $\left(u_{h}, \phi_{h}\right)$ in $\mathbb{X}_{h}$ such that

$$
\forall\left(w_{h}, \chi_{h}\right) \in \mathbb{X}_{h}, \quad a\left(u_{h}, \phi_{h} ; w_{h}, \chi_{h}\right)=g w_{h}(1)+\left(a \theta_{A}, \chi_{h}\right)
$$

It follows from the imbedding of $\mathbb{X}_{h}$ into $\mathbb{X}$ that the ellipticity property of the form $a(\cdot ; \cdot)$ established in Lemma 2.2 still holds on $\mathbb{X}_{h}$. This leads to the following result.

Proposition 3.1. For any constants $g$, $a$ and $\theta_{A}$, problem (3.2) admits a unique solution.

Another consequence of the ellipticity is the following version of the Strang's lemma:

$$
\left\|\left(u-u_{h}, \phi-\phi_{h}\right)\right\|_{\mathbb{X}} \leq c \inf _{\left(v_{h}, \psi_{h}\right) \in \mathbb{X}_{h}}\left\|\left(u-v_{h}, \phi-\psi_{h}\right)\right\|_{\mathbb{X}}
$$


So, the following result is easily derived from the approximation properties of the space $\mathbb{X}_{h}$, see [2, chap. IX, lemma 1.2] for instance.

Proposition 3.2. If the solution $(u, \phi)$ of problem (2.3) belongs to $H^{s+1}(I)^{2}$ for a real number $s, 0 \leq s \leq k$, the following a priori estimate holds between this solution and the solution of problem (3.2)

$$
\left\|u-u_{h}\right\|_{H^{1}(I)}+\left\|\phi-\phi_{h}\right\|_{H^{1}(I)} \leq c h^{s}\left(\|u\|_{H^{s+1}(I)}+\|\phi\|_{H^{s+1}(I)}\right) .
$$

Remark 3.3. Clearly, the results stated in Propositions 3.1 and 3.2 are still valid when the right-hand side of problem (2.3) and its analogue for problem (3.2) are replaced by

$$
g w(1)-\left(f, w_{x}\right)+\left(a \theta_{A}, \chi\right)
$$

for any function $f$ in $L^{2}(I)$.

\subsection{The full problem.}

There also, the discrete problem associated with problem (2.2) is constructed by the Galerkin method and is stated as follows

Find $\left(u_{h}, \phi_{h}\right)$ in $\mathbb{X}_{h}$ such that

$$
\begin{array}{ll}
\forall w_{h} \in \mathbb{Y}_{h}, \quad\left(u_{h x}, w_{h x}\right)-\left(\phi_{h}, w_{h x}\right)+\left(N\left(u_{h}\right) u_{h x}, w_{h x}\right)=g\left(u_{h}\right)(1) w_{h}(1), \\
\forall \chi_{h} \in \mathbb{Y}_{h}, \quad\left(\phi_{h x}, \chi_{h x}\right)-\left(u_{h x}, \chi_{h}\right)+\left(\phi_{h}, \chi_{h}\right)=\left(a \theta_{A}, \chi_{h}\right) .
\end{array}
$$

The existence of a solution for problem (3.5) can be derived by the same arguments as in Section 2. However, the proof is slightly simpler here since $\mathbb{Y}_{h}$ is finite-dimensional.

Proposition 3.4. For all $\rho<\rho_{0}$ and $\beta>\beta_{0}$ and all data $g_{1}, g_{2}$, a and $\theta_{A}$, problem (3.5) admits at least a solution. Moreover, for all $\beta>-\alpha$, this solution is unique and satisfies $(2.4)$.

To prove an a priori estimate, we deduce from the conformity of the discretization that problem $(2.2)$ can be written with $(w, \chi)$ replaced by any pair $\left(w_{h}, \chi_{h}\right)$ in $\mathbb{X}_{h}$. Subtracting problem (3.5) from this, we obtain

$$
a\left(u-u_{h}, \phi-\phi_{h} ; w_{h}, \chi_{h}\right)+\left(N(u) u_{x}-N\left(u_{h}\right) u_{h x}, w_{h x}\right)-\left(g(u)(1)-g\left(u_{h}(1)\right) w_{h}(1)=0 .\right.
$$

Thanks to this equation, we are in a position to prove a first error estimate. 
Proposition 3.5. For all $\rho<\rho_{0}$ and $\beta>\beta_{0}$, if the solution $(u, \phi)$ of problem (2.2) belongs to $H^{s+1}(I)^{2}$ for a real number $s, 0 \leq s \leq k$, the following a priori estimate holds between this solution and the solution of problem (3.5)

$$
\left\|u-u_{h}\right\|_{H^{1}(I)}+\left\|\phi-\phi_{h}\right\|_{H^{1}(I)} \leq c h^{s}\left(\|u\|_{H^{s+1}(I)}+\|\phi\|_{H^{s+1}(I)}\right) .
$$

Proof. Let $v_{h}$ and $\psi_{h}$ be the Lagrange interpolates of $u$ and $\phi$, respectively. By applying equation (3.6) with $w_{h}=u_{h}-v_{h}$ and $\chi_{h}=\phi_{h}-\psi_{h}$, we derive

$$
\begin{aligned}
a\left(u-u_{h}, \phi-\phi_{h}\right. & \left.; u-u_{h}, \phi-\phi_{h}\right)=a\left(u-u_{h}, \phi-\phi_{h} ; u-v_{h}, \phi-\psi_{h}\right) \\
& +\left(N(u) u_{x}-N\left(u_{h}\right) u_{h x}, u_{h x}-v_{h x}\right)-\left(g(u)(1)-g\left(u_{h}(1)\right)\left(u_{h}-v_{h}\right)(1) .\right.
\end{aligned}
$$

Owing to the choice of $v_{h}, v_{h}(1)$ is equal to $u(1)$, so that Lemma 2.6 implies that the quantity $-\left(g(u)(1)-g\left(u_{h}(1)\right)\left(u_{h}-v_{h}\right)(1)\right.$ is nonpositive. Combined with the ellipticity of $a(\cdot ; \cdot)$ established in Lemma 2.2 , this yields

$$
\begin{aligned}
\alpha\left\|\left(u-u_{h}, \phi-\phi_{h}\right)\right\|_{\mathbb{X}}^{2} \leq a\left(u-u_{h}, \phi-\phi_{h} ; u\right. & \left.-\boldsymbol{v}_{h}, \phi-\psi_{h}\right) \\
& +\left(N(u) u_{x}-N\left(u_{h}\right) u_{h x}, u_{h x}-v_{h x}\right) .
\end{aligned}
$$

The continuity of the form $a(\cdot, \cdot)$ leads to estimate the first term. To handle the second one, we oberve that

$$
\begin{aligned}
& \left(N(u) u_{x}-N\left(u_{h}\right) u_{h x}, u_{h x}-v_{h x}\right) \\
& \quad=\beta\left(u_{x}-u_{h x}, u_{h x}-v_{h x}\right)+\rho\left(|u|_{H^{1}(I)}^{2} u_{x}-\left|u_{h x}\right|_{H^{1}(I)}^{2} u_{h x}, u_{h x}-v_{h x}\right) .
\end{aligned}
$$

We now estimate successively the two terms in the right-hand side.

1) We observe that

$$
\beta\left(u_{x}-u_{h x}, u_{h x}-v_{h x}\right)=\beta\left(u_{x}-u_{h x}, u_{h x}-u_{x}\right)+\beta\left(u_{x}-u_{h x}, u_{x}-v_{h x}\right) .
$$

When $\beta$ is positive, the first term is negative and the second one is bounded thanks to a Cauchy-Schwarz inequality. When $\beta$ is negative, the first term is transported in the right-hand side of equation (3.8). The left-hand of this equation thus becomes

$$
(\alpha+\beta)\left\|u-u_{h}\right\|_{H^{1}(I)}^{2}+\alpha\left\|\phi-\phi_{h}\right\|_{H^{1}(I)}^{2}
$$

and since $\beta>\beta_{0}, \alpha+\beta$ is positive. The second term is there also evaluated thanks to a triangle inequality.

2)The last term is treated by using triangle inequalities and noting that, since $\beta>\beta_{0}$, both $u$ and $u_{h}$ are bounded in $H^{1}(I)$. This gives

$$
\rho\left(|u|_{H^{1}(I)}^{2} u_{x}-\left|u_{h x}\right|_{H^{1}(I)}^{2} u_{h x}, u_{h x}-v_{h x}\right) \leq c \rho\left|u-u_{h}\right|_{H^{1}(I)}\left|u_{h}-v_{h^{\prime}}\right|_{H^{1}(I)} .
$$

There, we use the fact that $\rho<\rho_{0}$ to conclude.

Combining all this leads to

$$
\left\|\left(u-u_{h}, \phi-\phi_{h}\right)\right\|_{\mathbb{X}} \leq c\left\|\left(u-v_{h}, \phi-\psi_{h}\right)\right\|_{\mathbb{X}} .
$$

The approximation properties of the space $\mathbb{X}_{h}$, see once more [2, chap. IX, lemma 1.2], thus give the desired result.

Even if the limitations on $\beta$ and $\rho$ are a little restrictive, estimate (3.7) is fully optimal. 


\section{A posteriori analysis of the discretization.}

Our a posteriori estimates rely on the residual equation satisfied by the discrete solution $\left(u_{h}, \phi_{h}\right)$. As previously, we write this equation successively in the linear and nonlinear cases.

\subsection{The linear problem.}

Once more, we take $N(u)$ equal to zero and $g(u)(1)$ equal to a constant $g$. By subtracting problem (3.2) from problem (2.3), we observe that, for any $(w, \chi)$ in $\mathbb{X}$ and when taking $w_{h}$ and $\chi_{h}$ equal to the Lagrange interpolate of $w$ and $\chi$ (so that $w_{h}(1)$ is equal to $w(1))$

$$
a\left(u-u_{h}, \phi-\phi_{h} ; w, \chi\right)=\left(a \theta_{A}, \chi-\chi_{h}\right)-a\left(u_{h}, \phi_{h} ; w-w_{h}, \chi-\chi_{h}\right) .
$$

By integrating by parts on each $K$ in $\mathcal{T}_{h}$ and noting that both $w-w_{h}$ and $\chi-\chi_{h}$ vanish at all endpoints of elements $K$, we obtain

$$
\begin{aligned}
a\left(u-u_{h}, \phi-\phi_{h} ; w, \chi\right) & =\sum_{K \in \mathcal{T}_{h}}\left(\int_{K}\left(u_{h x x}-\phi_{h x}\right)(x)\left(w-w_{h}\right)(x) d x\right. \\
& \left.+\int_{K}\left(a \theta_{A}+\phi_{h x x}+u_{h x}-\phi_{h}\right)(x)\left(\chi-\chi_{h}\right)(x) d x .\right)
\end{aligned}
$$

The definition of the error indicators follows from this last equation in a natural way.

Definition 4.1. Each error indicator $\eta_{K}, K \in \mathcal{T}_{h}$, is defined by

$$
\eta_{K}=h_{K}\left\|u_{h x x}-\phi_{h x}\right\|_{L^{2}(K)}+h_{K}\left\|a \theta_{A}+\phi_{h x x}+u_{h x}-\phi_{h}\right\|_{L^{2}(K)} .
$$

It must be noted that these indicators can be computed easily since the norms only involves polynomials. Moreover, they are of residual type: when suppressing the indices $h$, everything vanishes, see problem (2.1). Moreover, it is very easy to bound the error as a function of these indicators.

Proposition 4.2. The following a posteriori error estimate holds between the solution $(u, \phi)$ of problem $(2.3)$ and the solution $\left(u_{h}, \phi_{h}\right)$ of problem $(3.2)$

$$
\left\|u-u_{h}\right\|_{H^{1}(I)}+\left\|\phi-\phi_{h}\right\|_{H^{1}(I)} \leq c\left(\sum_{K \in \mathcal{T}_{h}} \eta_{K}^{2}\right)^{\frac{1}{2}} .
$$

Proof. By using the ellipticity of $a(\cdot ; \cdot)$, see Lemma 2.2, and a Cauchy-Schwarz inequality, we deduce from equation (4.1) that

$$
\begin{aligned}
\alpha\left\|\left(u-u_{h}, \phi-\phi_{h}\right)\right\|_{\mathbb{X}}^{2} \leq \sum_{K \in \mathcal{T}_{h}}( & \left\|u_{h x x}-\phi_{h x}\right\|_{L^{2}(K)}\left\|w-w_{h}\right\|_{L^{2}(K)} \\
& \left.+\left\|a \theta_{A}+\phi_{h x x}+u_{h x}-\phi_{h}\right\|_{L^{2}(K)}\left\|\chi-\chi_{h}\right\|_{L^{2}(K)}\right),
\end{aligned}
$$


with $w=u$ and $\chi=\phi$. So the desired estimate is easily obtained from the basic approximation properties of the Lagrange interpolation operator, see [2, chap. IX, lemma $1.2]$.

Remark 4.3. The constant $c$ on the previous estimate mainly depends on the norm of the interplation operator on a reference element, hence is easy to compute. This is of great interest when the a posteriori error estimates are used for security criteria.

On the other hand, it can be noted that the residual equation (4.1) can also be written, for any functions $w$ and $\chi$ vanishing at all endpoints of the elements $K$ of $\mathcal{T}_{h}$,

$$
\begin{aligned}
a\left(u-u_{h}, \phi-\phi_{h} ; w, \chi\right) & =\sum_{K \in \mathcal{T}_{h}}\left(\int_{K}\left(u_{h x x}-\phi_{h x}\right)(x) w(x) d x\right. \\
& \left.+\int_{K}\left(a \theta_{A}+\phi_{h x x}+u_{h x}-\phi_{h}\right)(x) \chi(x) d x .\right)
\end{aligned}
$$

This leads to the upper bounds for the indicators.

Proposition 4.4. Each indicator $\eta_{K}, K \in \mathcal{T}_{h}$, defined in (4.2), satisfies

$$
\eta_{K} \leq c\left(\left\|u-u_{h}\right\|_{H^{1}(K)}+\left\|\phi-\phi_{h}\right\|_{H^{1}(K)}\right) .
$$

Proof. We proceed in two steps, bounding separately each term in the indicator.

1) In equation (4.4), we first take $w$ equal to $w_{K}$ and $\chi$ equal to zero, with

$$
w_{K}= \begin{cases}\left(u_{h x x}-\phi_{h x}\right) \psi_{K} & \text { on } K \\ 0 & \text { elsewhere }\end{cases}
$$

where $\psi_{K}$ is the bubble function on $K$ (equal to the product of the two barycentric coordinates associated with the endpoints of $K$ ). This yields

$$
\left\|\left(u_{h x x}-\phi_{h x}\right) \psi_{K}^{\frac{1}{2}}\right\|_{L^{2}(K)}^{2} \leq c\left(\left\|u-u_{h}\right\|_{H^{1}(K)}+\left\|\phi-\phi_{h}\right\|_{H^{1}(K)}\right)\left\|w_{K}\right\|_{H^{1}(K)} .
$$

We then use the inverse inequalities, valid for any polynomial $z$ of fixed degree, see e.g. [11, Proposition 3.37],

$$
\|z\|_{L^{2}(K)} \leq c\left\|z \psi_{K}^{\frac{1}{2}}\right\|_{L^{2}(K)}, \quad\|z\|_{H^{1}(K)} \leq c^{\prime} h_{K}^{-1}\|z\|_{L^{2}(K)} .
$$

All this gives

$$
h_{K}\left\|u_{h x x}-\phi_{h x}\right\|_{L^{2}(K)} \leq c\left(\left\|u-u_{h}\right\|_{H^{1}(K)}+\left\|\phi-\phi_{h}\right\|_{H^{1}(K)}\right) .
$$

2) Similarly, we take in equation (4.4) $w$ equal to 0 and $\chi$ equal to $\chi_{K}$, with

$$
\chi_{K}= \begin{cases}\left(a \theta_{A}+\phi_{h x x}+u_{h x}-\phi_{h}\right) \psi_{K} & \text { on } K, \\ 0 & \text { elsewhere. }\end{cases}
$$


Exactly the same arguments as previously yield

$$
h_{K}\left\|a \theta_{A}+\phi_{h x x}+u_{h x}-\phi_{h}\right\|_{L^{2}(K)} \leq c\left(\left\|u-u_{h}\right\|_{H^{1}(K)}+\left\|\phi-\phi_{h}\right\|_{H^{1}(K)}\right) .
$$

Estimate (4.5) is a direct consequence of (4.7) and (4.8).

\subsection{The full problem.}

The residual equation is a little more complex in this case. For brevity, we introduce the nonlinear form

$$
\mathcal{A}(u, \phi ; w, \chi)=a(u, \phi ; w, \chi)+\left(N(u) u_{x}, w_{x}\right)-g(u)(1) w(1) .
$$

Thus, the residual equation can be written as

$$
\mathcal{A}(u, \phi ; w, \chi)-\mathcal{A}\left(u_{h}, \phi_{h} ; w, \chi\right)=\left(a \theta_{A}, \chi-\chi_{h}\right)-\mathcal{A}\left(u_{h}, \phi_{h} ; w-w_{h}, \chi-\chi_{h}\right),
$$

where we now take $w_{h}$ and $\chi_{h}$ equal to the Lagrange interpolate of $w$ and $\chi$, respectively. By noting that $\left(w-w_{h}\right)(1)$ is equal to zero and integrating by parts on each $K$ in $\mathcal{T}_{h}$, we obtain

$$
\begin{aligned}
& \mathcal{A}(u, \phi ; w, \chi)-\mathcal{A}\left(u_{h}, \phi_{h} ; w, \chi\right) \\
& =\sum_{K \in \mathcal{T}_{h}}\left(\int_{K}\left(u_{h x x}-\phi_{h x}+N\left(u_{h}\right) u_{h x x}\right)(x)\left(w-w_{h}\right)(x) d x\right. \\
& \left.\quad+\int_{K}\left(a \theta_{A}+\phi_{h x x}+u_{h x}-\phi_{h}\right)(x)\left(\chi-\chi_{h}\right)(x) d x\right) .
\end{aligned}
$$

There also, this leads to the definition of the indicators in a natural way.

Definition 4.5. Each error indicator $\eta_{K}, K \in \mathcal{T}_{h}$, is defined by

$$
\eta_{K}=h_{K}\left\|u_{h x x}-\phi_{h x}+N\left(u_{h}\right) u_{h x x}\right\|_{L^{2}(K)}+h_{K}\left\|a \theta_{A}+\phi_{h x x}+u_{h x}-\phi_{h}\right\|_{L^{2}(K)} .
$$

Despite the nonlinear term, these indicators are still easy to compute. We need a further lemma before proving the first estimate.

Lemma 4.6. For $\beta>\beta_{0}$, the form $\mathcal{A}(\cdot, \cdot)$ satisfies the following property, for a positive constant $\alpha_{*}$, for all pairs $\left(u_{1}, \phi_{1}\right)$ and $\left(u_{2}, \phi_{2}\right)$ in $\mathbb{X}$,

$$
\mathcal{A}\left(u_{1}, \phi_{1} ; u_{1}-u_{2}, \phi_{1}-\phi_{2}\right)-\mathcal{A}\left(u_{2}, \phi_{2} ; u_{1}-u_{2}, \phi_{1}-\phi_{2}\right) \geq \alpha^{*}\left\|\left(u_{1}-u_{2}, \phi_{1}-\phi_{2}\right)\right\|_{\mathbb{X}}^{2} \text {. }
$$

Proof. By using Lemma 2.2, we have

$$
\begin{gathered}
\mathcal{A}\left(u_{1}, \phi_{1} ; u_{1}-u_{2}, \phi_{1}-\phi_{2}\right)-\mathcal{A}\left(u_{2}, \phi_{2} ; u_{1}-u_{2}, \phi_{1}-\phi_{2}\right) \\
\geq(\alpha+\beta)\left\|u_{1}-u_{2}\right\|_{H^{1}(I)}^{2}+\alpha\left\|\phi_{1}-\phi_{2}\right\|_{H^{1}(I)}^{2}-\left(g\left(u_{1}\right)(1)-g\left(u_{2}\right)(1)\right)\left(u_{1}-u_{2}\right)(1) \\
+N_{u_{1}}^{*}\left(u_{1}\right)\left(u_{1 x}, u_{1 x}-u_{2 x}\right)-N_{u_{2}}^{*}\left(u_{2}\right)\left(u_{2 x}, u_{1 x}-u_{2 x}\right) .
\end{gathered}
$$


So the desired result follows by applying formula (2.7) and Lemma 2.6 to handle the nonlinear terms.

Applying Lemma 4.6 in equation (4.9) (with obvious choice of $\left(u_{1}, \phi_{1}\right)$ and $\left(u_{2}, \phi_{2}\right)$ ) and using exactly the same arguments as in the proof of Proposition 4.2 leads to the following statement.

Proposition 4.7. The following a posteriori error estimate holds between the solution $(u, \phi)$ of problem $(2.2)$ and the solution $\left(u_{h}, \phi_{h}\right)$ of problem (3.5)

$$
\left\|u-u_{h}\right\|_{H^{1}(I)}+\left\|\phi-\phi_{h}\right\|_{H^{1}(I)} \leq c\left(\sum_{K \in \mathcal{T}_{h}} \eta_{K}^{2}\right)^{\frac{1}{2}} .
$$

On the other hand, equation (4.9) can also be written, for any functions $w$ and $\chi$ vanishing at all endpoints of the elements $K$ of $\mathcal{T}_{h}$,

$$
\begin{gathered}
\mathcal{A}(u, \phi ; w, \chi)-\mathcal{A}\left(u_{h}, \phi_{h} ; w, \chi\right)=\sum_{K \in \mathcal{T}_{h}}\left(\int_{K}\left(u_{h x x}-\phi_{h x}+N\left(u_{h}\right) u_{h x x}\right)(x) w(x) d x\right. \\
\left.+\int_{K}\left(a \theta_{A}+\phi_{h x x}+u_{h x}-\phi_{h}\right)(x) \chi(\boldsymbol{x}) d \boldsymbol{x}\right) .
\end{gathered}
$$

This yields the upper bound for the indicator.

Proposition 4.8. Each indicator $\eta_{K}, K \in \mathcal{T}_{h}$, defined in (4.10), satisfies

$$
\eta_{K} \leq c\left(\left\|u-u_{h}\right\|_{H^{1}(K)}+\left\|\phi-\phi_{h}\right\|_{H^{1}(K)}\right) .
$$

Proof. In equation (4.13), we take successively $(w, \chi)$ equal to $\left(\tilde{w}_{K}, 0\right)$ and to $\left(0, \chi_{K}\right)$, where the function $\tilde{w}_{K}$ is defined by

$$
\tilde{w}_{K}= \begin{cases}\left(u_{h x x}-\phi_{h x}+N\left(u_{h}\right) u_{h x x}\right) \psi_{K} & \text { on } K \\ 0 & \text { elsewhere }\end{cases}
$$

while the function $\chi_{K}$ is exactly the same as in the proof of Proposition 4.4. The same arguments as in this proof combined with the continuity property (which requires the boundedness of $u$ and $u_{h}$, see Lemma 2.4 and Proposition 3.4)

$$
\mathcal{A}(u, \phi ; w, \chi)-\mathcal{A}\left(u_{h}, \phi_{h} ; w, \chi\right) \leq c\left\|\left(u-u_{h}, \phi-\phi_{h}\right)\right\|_{\mathbb{X}}\|(w, \chi)\|_{\mathbb{X}},
$$

yield the desired estimate.

When comparing estimates (4.12) and (4.14), we observe that the error is equivalent to the Hilbertian sum of the error indicators, so that our estimates are fully optimal. Moreover, estimate (4.14) is local, so that the proposed indicators are an efficient tool for mesh adaptivity. We do not perform adaptivity in the next computations since we only work with very smooth solutions. 


\section{An iterative algorithm.}

Since problem (3.5) is nonlinear, we now propose an algorithm for solving it. We start from a given solution $\left(u^{0}, \phi^{0}\right)$ (for instance, the solution of the linear problem (3.2) or an approximation of it) and, assuming that $\left(u_{h}^{n-1}, \phi_{h}^{n-1}\right)$ is known, we look for a solution of the following system

Find $\left(u_{h}^{n}, \phi_{h}^{n}\right)$ in $\mathbb{X}_{h}$ such that

$$
\begin{array}{ll}
\forall w_{h} \in \mathbb{Y}_{h}, & \left(u_{h x}^{n}, w_{h x}\right)-\left(\phi_{h}^{n}, w_{h x}\right)+\left(N\left(u_{h}^{n-1}\right) u_{h x}^{n}, w_{h x}\right)=g\left(u_{h}^{n-1}\right)(1) w_{h}(1), \\
\forall \chi_{h} \in \mathbb{Y}_{h}, & \left(\phi_{h x}^{n}, \chi_{h x}\right)+\left(\phi_{h}^{n}, \chi_{h}\right)=\left(a \theta_{A}, \chi_{h}\right)+\left(u_{h x}^{n-1}, \chi_{h}\right) .
\end{array}
$$

Since this problem is fully linear, proving the existence and the uniqueness of its solution follows exactly the same lines as for Proposition 3.1. Moreover, the two equations in (5.1) are fully uncoupled.

In a first step, we prove a bound for the part $u_{h}^{n}$ of the solution of this problem.

Lemma 5.1. For all $\beta>-\alpha$, the part $u_{h}^{n}, n \geq 1$, of the solution of problem (5.1) satisfies, for a constant $c_{*}$ independent of $h$ and $n$

$$
\left|u_{h}^{n}\right|_{H^{1}(I)} \leq c_{*}\left(\left|u_{h}^{n}-u_{h}^{n-1}\right|_{H^{1}(I)}+\left|a \theta_{A}\right|\right) .
$$

Proof: Equation $(5.1)$ can be written, forall $\left(w_{h}, \chi_{h}\right)$ in $\mathbb{X}_{h}$,

$$
\begin{aligned}
a\left(u_{h}^{n}, \phi_{h}^{n} ; w_{h} ; \chi_{h}\right) & +\left(N\left(u_{h}^{n-1}\right) u_{h x}^{n}, w_{h x}\right)-g\left(u_{h}^{n}\right)(1) w_{h}(1) \\
& =\left(g\left(u_{h}^{n-1}\right)(1)-g\left(u_{h}^{n}(1)\right) w_{h}(1)+\left(a \theta_{A}, \chi_{h}\right)+\left(u_{h x}^{n-1}-u_{h x}^{n}, \chi_{h}\right) .\right.
\end{aligned}
$$

Thus, by taking $\left(w_{h}, \chi_{h}\right)$ equal to $\left(u_{h}^{n}, \phi_{h}^{n}\right)$ and combining Lemma 2.2 with the positivity properties of $N$ and the fact that $-g\left(u_{h}^{n}\right)(1) u_{h}^{n}(1)$ is nonnegative, we derive

$$
\begin{aligned}
(\alpha+\beta)\left|u_{h}^{n}\right|_{H^{1}(I)}^{2} & +\alpha\left|\phi_{h}^{n}\right|_{H^{1}(I)}^{2} \\
& \leq \mid g\left(u_{h}^{n-1}\right)(1)-g\left(\left.u_{h}^{n}(1)|| u_{h}^{n}(1)\left|+\left(\left|a \theta_{A}\right|+c\left|u_{h}^{n-1}-u_{h}^{n}\right|_{H^{1}(I)}\right)\right| \phi_{h}^{n}\right|_{H^{1}(I)}\right.
\end{aligned},
$$

where the constant $c$ here comes from a Poincaré-Friedrichs inequality. We conclude thanks to the Lipschitz continuity of $g$.

We now set: $\left[u_{h}\right]^{n}=u_{h}^{n}-u_{h}^{n-1}$ and $\left[\phi_{h}\right]^{n}=\phi_{h}^{n}-\phi_{h}^{n-1}$. Subtracting problem (5.1) at step $n-1$ from problem (5.1) at step $n$, we derive

$$
\begin{array}{ll}
\forall w_{h} \in \mathbb{Y}_{h}, \quad\left(\left[u_{h}\right]_{x}^{n}, w_{h x}\right)-\left(\left[\phi_{h}\right]^{n}, w_{h x}\right) & \\
& +\left(N\left(u_{h}^{n-1}\right) u_{h x}^{n}-N\left(u_{h}^{n-2}\right) u_{h x}^{n-1}, w_{h x}\right)=\left(g\left(u_{h}^{n-1}\right)-g\left(u_{h}^{n-2}\right)\right)(1) w_{h}(1), \\
\forall \chi_{h} \in \mathbb{Y}_{h}, \quad\left(\left[\phi_{h}\right]_{x}^{n}, \chi_{h x}\right)+\left(\left[\phi_{h}\right]^{n}, \chi_{h}\right)=\left(\left[u_{h}\right]_{x}^{n-1}, \chi_{h}\right) .
\end{array}
$$


We are thus in a position to prove the key lemma in this section.

Lemma 5.2. There exists two real numbers $\rho_{0}^{*}>0$ and $\eta, 0<\eta<1$, such that the following estimate holds for $\beta>\frac{1}{\varepsilon}$ and $\rho<\rho_{0}^{*}$, for all $n \geq 2$

$$
\left|\left[u_{h}\right]^{n}\right|_{H^{1}(I)} \leq \eta\left|\left[u_{h}\right]^{n-1}\right|_{H^{1}(I)} .
$$

Proof: First, by taking $\chi_{h}$ equal to $\left[\phi_{h}\right]^{n}$ in the second line of (5.3) yields

$$
\left|\left[\phi_{h}\right]^{n}\right|_{H^{1}(I)}^{2}+\frac{1}{2}\left\|\left[\phi_{h}\right]^{n}\right\|_{L^{2}(I)}^{2} \leq \frac{1}{2}\left|\left[u_{h}\right]^{n-1}\right|_{H^{1}(I)}^{2} .
$$

It follows from the Poincaré-Friedrichs inequality that

$$
\left(\frac{1}{2}+\frac{1}{c}\right)\left\|\left[\phi_{h}\right]^{n}\right\|_{L^{2}(I)}^{2} \leq \frac{1}{2}\left|\left[u_{h}\right]^{n-1}\right|_{H^{1}(I)}^{2} .
$$

Next, setting $N^{*}(u)=N_{u}^{*}(u)$ for brevity and taking $w_{h}$ equal to $\left[u_{h}\right]^{n}$ gives

$$
\begin{gathered}
(1+\beta)\left|\left[u_{h}\right]^{n}\right|_{H^{1}(I)}^{2} \leq \\
\left\|\left[\phi_{h}\right]^{n}\right\|_{L^{2}(I)}\left|\left[u_{h}\right]^{n}\right|_{H^{1}(I)}+\left|\left(g\left(u_{h}^{n-1}\right)-g\left(u_{h}^{n-2}\right)\right)(1)\right|\left|\left[u_{h}\right]^{n}(1)\right| \\
+\left\|N^{*}\left(u_{h}^{n-1}\right) u_{h x}^{n}-N^{*}\left(u_{h}^{n-2}\right) u_{h x}^{n-1}\right\|_{L^{2}(I)}\left|\left[u_{h}\right]^{n}\right|_{H^{1}(I)} .
\end{gathered}
$$

We thus simplify by $\left|\left[u_{h}\right]^{n}\right|_{H^{1}(I)}$. To go further,

1 ) we use (5.5) to bound the first term in the right-hand side, whence the constant $c_{0}=$ $\left(1+\frac{2}{c}\right)^{-\frac{1}{2}}$

2) we observe that the function $g$ is Lipschitz-continuous with Lipschitz constant $\frac{1}{\varepsilon}$, see the proof of Lemma 2.6;

3 ) we observe that the nonlinearity $N^{*}$ is also Lipschitz-continuous and we use a triangle inequality. Combined with Lemma 5.1, this yields

$$
\begin{aligned}
& \left\|N^{*}\left(u_{h}^{n-1}\right) u_{h x}^{n}-N^{*}\left(u_{h}^{n-2}\right) u_{h x}^{n-1}\right\|_{L^{2}(I)} \\
& \quad \leq\left\|N^{*}\left(u_{h}^{n-1}\right)\left[u_{h x}\right]^{n}\right\|_{L^{2}(I)}+\left\|\left(N^{*}\left(u_{h}^{n-1}\right)-N^{*}\left(u_{h}^{n-2}\right)\right) u_{h x}^{n-1}\right\|_{L^{2}(I)} \\
& \quad \leq \rho c_{*}^{2}\left(\left|\left[u_{h}\right]^{n-1}\right|_{H^{1}(I)}+\left|a \theta_{A}\right|\right)^{2}\left|\left[u_{h}\right]^{n}\right|_{H^{1}(I)} \\
& +\rho c_{*}\left(\left|\left[u_{h}\right]^{n-1}\right|_{H^{1}(I)}+\left|a \theta_{A}\right|\right) c_{*}\left(\left|\left[u_{h}\right]^{n-1}\right|_{H^{1}(I)}+\left|\left[u_{h}\right]^{n-2}\right|_{H^{1}(I)}+2\left|a \theta_{A}\right|\right)\left|\left[u_{h}\right]^{n-1}\right|_{H^{1}(I)} .
\end{aligned}
$$

All this gives

$$
\left(1+\beta-c_{1} \rho\right)\left|\left[u_{h}\right]^{n}\right|_{H^{1}(I)} \leq\left(c_{0}+\frac{1}{\varepsilon}+c_{2} \rho\right)\left|\left[u_{h}\right]^{n-1}\right|_{H^{1}(I)},
$$

where the constants $c_{1}$ and $c_{2}$ are defined by

$$
\begin{aligned}
& c_{1}=c_{*}^{2}\left(\left|\left[u_{h}\right]^{n-1}\right|_{H^{1}(I)}+\left|a \theta_{A}\right|\right)^{2}, \\
& \quad c_{2}=c_{*}\left(\left|\left[u_{h}\right]^{n-1}\right|_{H^{1}(I)}+\left|a \theta_{A}\right|\right) c_{*}\left(\left|\left[u_{h}\right]^{n-1}\right|_{H^{1}(I)}+\left|\left[u_{h}\right]^{n-2}\right|_{H^{1}(I)}+2\left|a \theta_{A}\right|\right) .
\end{aligned}
$$


Next, we assume by induction that the sequence $\left(u_{h}^{m}\right)_{1 \leq m \leq n-1}$ satisfies

$$
\left|\left[u_{h}\right]^{m}\right|_{H^{1}(I)} \leq\left|\left[u_{h}\right]^{1}\right|_{H^{1}(I)}, \quad 1 \leq m \leq n-1 .
$$

This yields the desired estimate (5.4) when $\rho$ is smaler than a constant $\rho_{0}^{*}$ independent of $n$ and also the induction hypothesis for $m=n$.

Even if the conditions on $\beta$ and $\rho$ are a little restrictive, the convergence of the algorithm is a direct consequence of the previous lemma.

Theorem 5.3. For $\beta>\frac{1}{\varepsilon}$ and $\rho<\rho_{0}^{*}$, the sequence $\left(u_{h}^{n}, \phi_{h}^{n}\right)$ defined from (5.1) converges to the solution $\left(u_{h}, \phi_{h}\right)$ of problem (3.5). Moreover, the following estimate holds

$$
\left\|u_{h}-u_{h}^{n}\right\|_{H^{1}(I)}+\left\|\phi_{h}-\phi_{h}^{n}\right\|_{H^{1}(I)} \leq 2 \frac{\eta^{n}}{1-\eta}\left\|u_{h}^{1}-u_{h}^{0}\right\|_{H^{1}(I)},
$$

for the constant $\eta<1$ introduced in Lemma 5.2.

Proof: We proceed in two steps.

1) From Lemma 5.2, we deduce that, for any positive integers $n$ and $m$,

$$
\left\|u_{h}^{n+m}-u_{h}^{n}\right\|_{H^{1}(I)} \leq \eta^{n} \frac{1-\eta^{m}}{1-\eta}\left\|u_{h}^{1}-u_{h}^{0}\right\|_{H^{1}(I)} .
$$

Thus, $\left(u_{h}^{n}\right)_{n}$ is a Cauchy sequence in $H_{*}^{1}(I)$. This yields its convergence, and the first part of estimate (5.6) follows from the previous line.

2) In the proof of Lemma 5.2, we have checked that

$$
\left|\left[\phi_{h}\right]^{n}\right|_{H^{1}(I)}^{2} \leq \frac{1}{2}\left|\left[u_{h}\right]^{n-1}\right|_{H^{1}(I)}^{2}
$$

Thus, exactly the same arguments as previously yield the convergence of $\left(\phi_{h}^{n}\right)_{n}$ and the second part of estimate (5.6).

From the previous theorem, the convergence is geometric, hence very fast. But, in any case, the following question arises: When must we stop the iteration? To answer this question, we follow the approach in [5]. Using the same notation as in Section 4.2 for the form $\mathcal{A}(\cdot, \cdot)$ (and also as in the proof of Lemma 5.2 for $N^{*}$ ), we have the following residual equation, for all $\left(w_{h}, \chi_{h}\right)$ in $\mathbb{X}_{h}$,

$$
\begin{aligned}
\mathcal{A}\left(u_{h}, \phi_{h} ; w_{h}, \chi_{h}\right)-\mathcal{A}\left(u_{h}^{n}, \phi_{h}^{n} ; w_{h}, \chi_{h}\right)=-\left(u_{h x}^{n}-u_{h x}^{n-1}, \chi_{h}\right) \\
-\left(\left(N^{*}\left(u_{n}^{n}\right)-N^{*}\left(u_{n}^{n-1}\right)\right) u_{h x}^{n}, w_{h x}\right)+\left(g\left(u_{h}^{n}\right)-g\left(u_{h}^{n-1}\right)\right)(1) w_{h}(1) .
\end{aligned}
$$

This leads to the next result.

Proposition 5.4. For $\beta>\beta_{0}$, the following a posteriori error estimate holds

$$
\left\|u_{h}-u_{h}^{n}\right\|_{H^{1}(I)}+\left\|\phi_{h}-\phi_{h}^{n}\right\|_{H^{1}(I)} \leq c\left|u_{h}^{n}-u_{h}^{n-1}\right|_{H^{1}(I)} .
$$


Proof: Owing to Lemma 4.6; we derive from (5.7) that

$$
\begin{aligned}
\alpha^{*}\left(\left\|u_{h}-u_{h}^{n}\right\|_{H^{1}(I)}+\left\|\phi-\phi_{h}\right\|_{H^{1}(I)}\right) \leq\left\|u_{h x}^{n}-u_{h x}^{n-1}\right\|_{L^{2}(I)} \\
+\left|N^{*}\left(u_{n}^{n}\right)-N^{*}\left(u_{n}^{n-1}\right)\right|\left\|u_{h x}^{n}\right\|_{L^{2}(I)}+\left|\left(g\left(u_{h}^{n}\right)-g\left(u_{h}^{n-1}\right)\right)(1)\right| .
\end{aligned}
$$

As for Lemma 5.1, evaluating the last two terms relies on the Lipschitz-continuity of $g$ and $N^{*}$ and the fact that the sequence of solutions $\left(u_{h}^{n}\right)$ is bounded, see Lemmas 5.1 and 5.2.

The constant $c$ in the previous proposition can be evaluated more precisely:

$$
c=\left(\alpha^{*}\right)^{-1}\left(1+\frac{1}{\varepsilon}+\left|u_{h}^{n}\right|_{H^{1}(I)}\left(\left|u_{h}^{n}\right|_{H^{1}(I)}+\left|u_{h}^{n-1}\right|_{H^{1}(I)}\right)\right) .
$$

But, in any case, we stop to iterate when the quantity $\left|u_{h}^{n}-u_{h}^{n-1}\right|_{H^{1}(I)}$ is smaller than a given tolerance. 


\section{Numerical experiments.}

In all experiments, the coefficients of the equation are given by

$$
a=0.017, \quad \epsilon=1, \quad g_{1}=-0.01, \quad g_{2}=0.1 .
$$

Piecewise affine functions are considered (i.e. $k=1$ ) and a uniform mesh with 100 nodes is used.
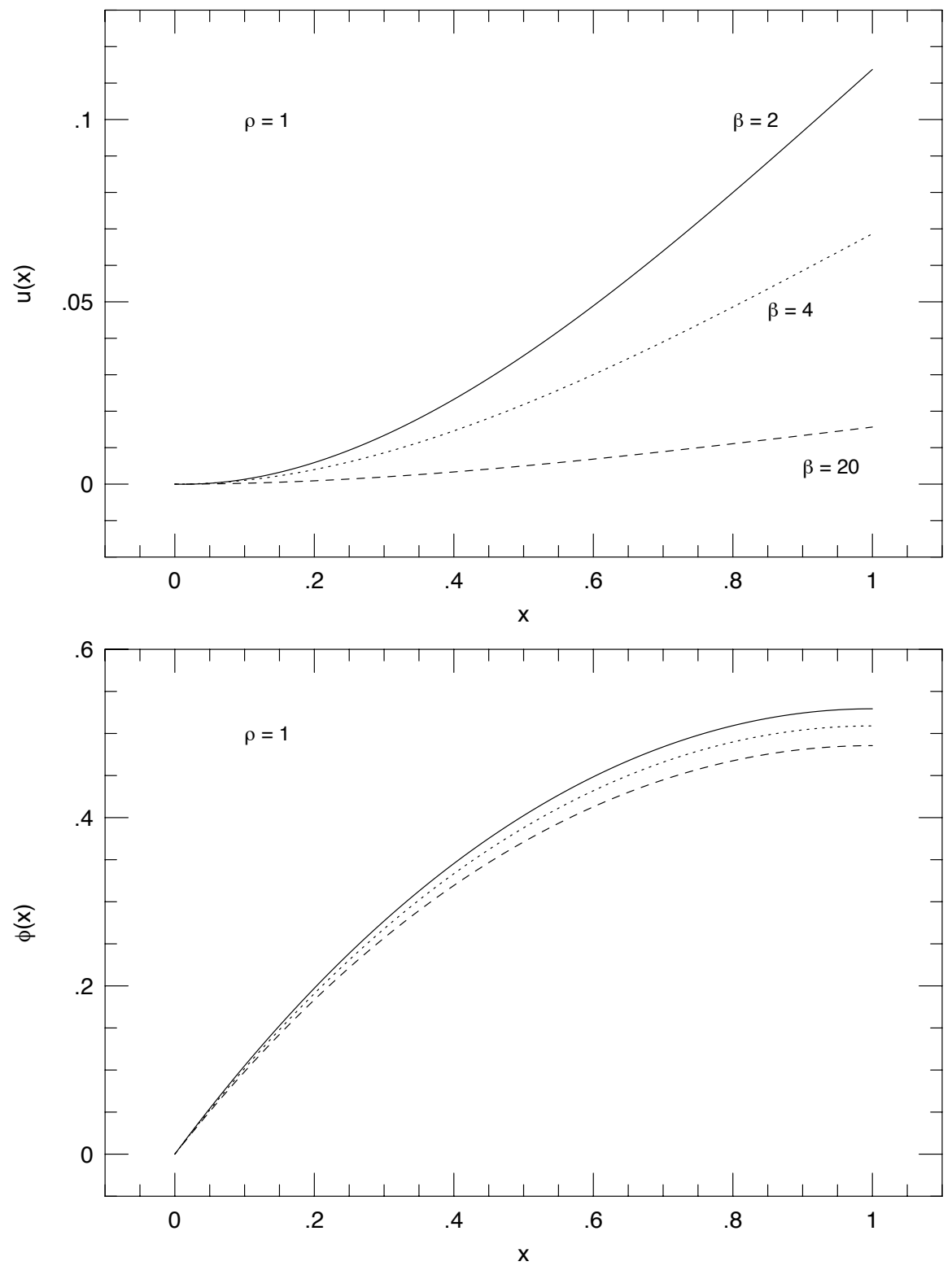

Figure 1. Vertical displacement and angular rotation when $\theta_{A}=80$ and $\rho=1$. 
All experiments are performed in the nonlinear case $\rho \neq 0$. We initialize the iterative algorithm with

$$
\begin{array}{r}
u_{h}^{0}(x)=\frac{1}{2}\left(\sigma(1)+a \theta_{A}\right)\left(x-\frac{1}{3}+\frac{(1-x)^{3}}{3}\right)+\sigma(1) x, \\
\phi_{h}^{0}=\frac{1}{2}\left(\sigma(1)+a \theta_{A}\right)\left(1-(1-x)^{2}\right),
\end{array}
$$

with $\sigma(1)=\frac{3 u(1)-a \theta_{A}}{3}$ for a given value of $u(1)$. Indeed, this is the solution of problem (2.1) when $\beta=\rho=0$. A tolerance of $10^{-7}$ is used to stop the iterative process.
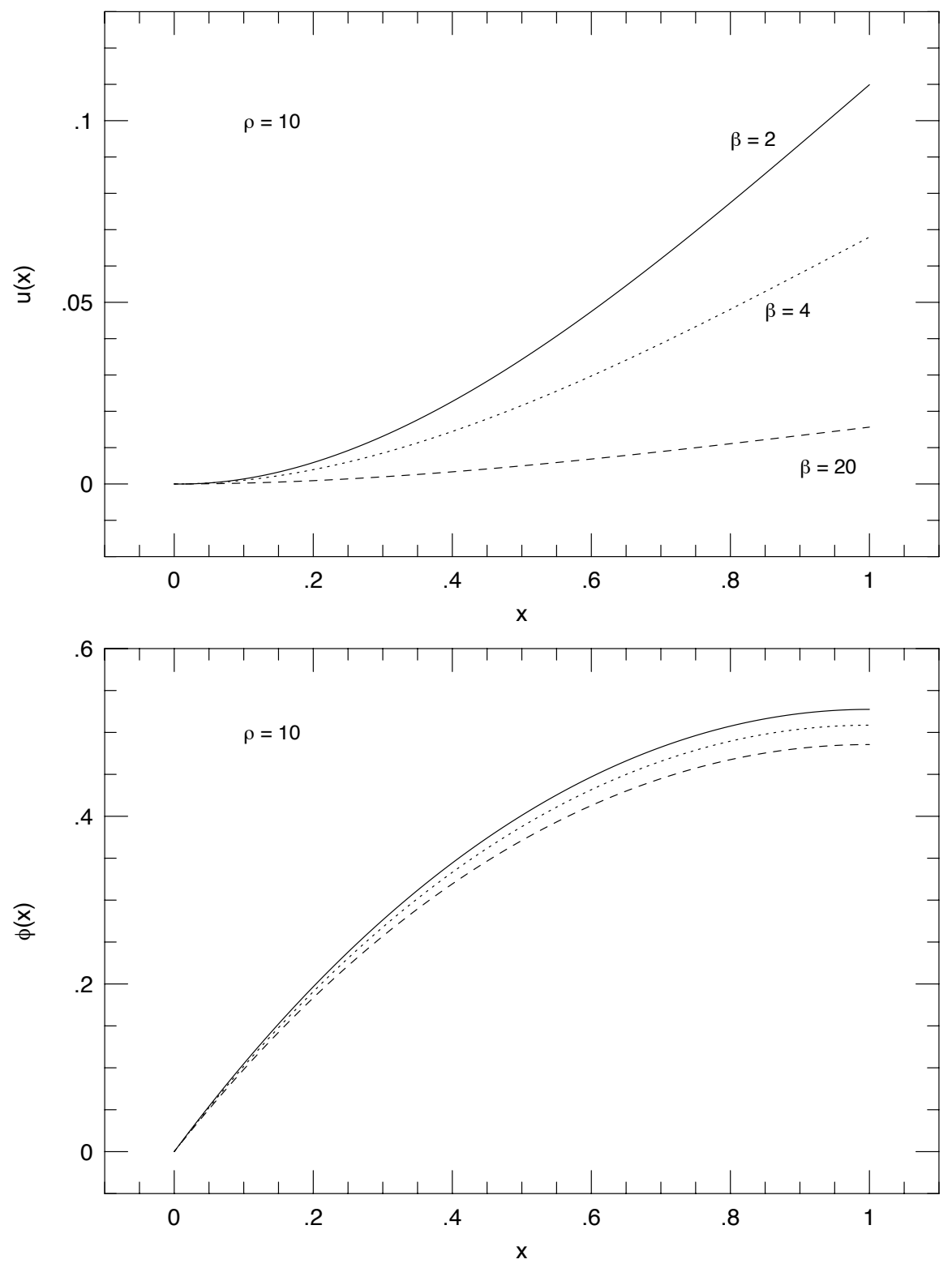

Figure 2. Vertical displacement and angular rotation when $\theta_{A}=80$ and $\rho=10$. 
In the first example the temperature at the endpoint $x=0$ is $\theta_{A}=80$. Initially, we choose $\rho=1$ and after, we set $\rho=10$. Figures 1 and 2 show the deformed configuration of the beam and the rotation angle for 3 different values of $\beta$. Contact with the upper obstacle is observed for $\beta=2$. No significant differences, apart from a slight movement of the displacement when $\beta=2$, are observed when we increase $\rho$.

In the second example, we changed the temperature of the left endpoint to $\theta_{A}=-20$. In this case and with $\rho=1$, contact with the lower obstacle is seen for $\beta=2$ and $\beta=4$ (see Figure 3).
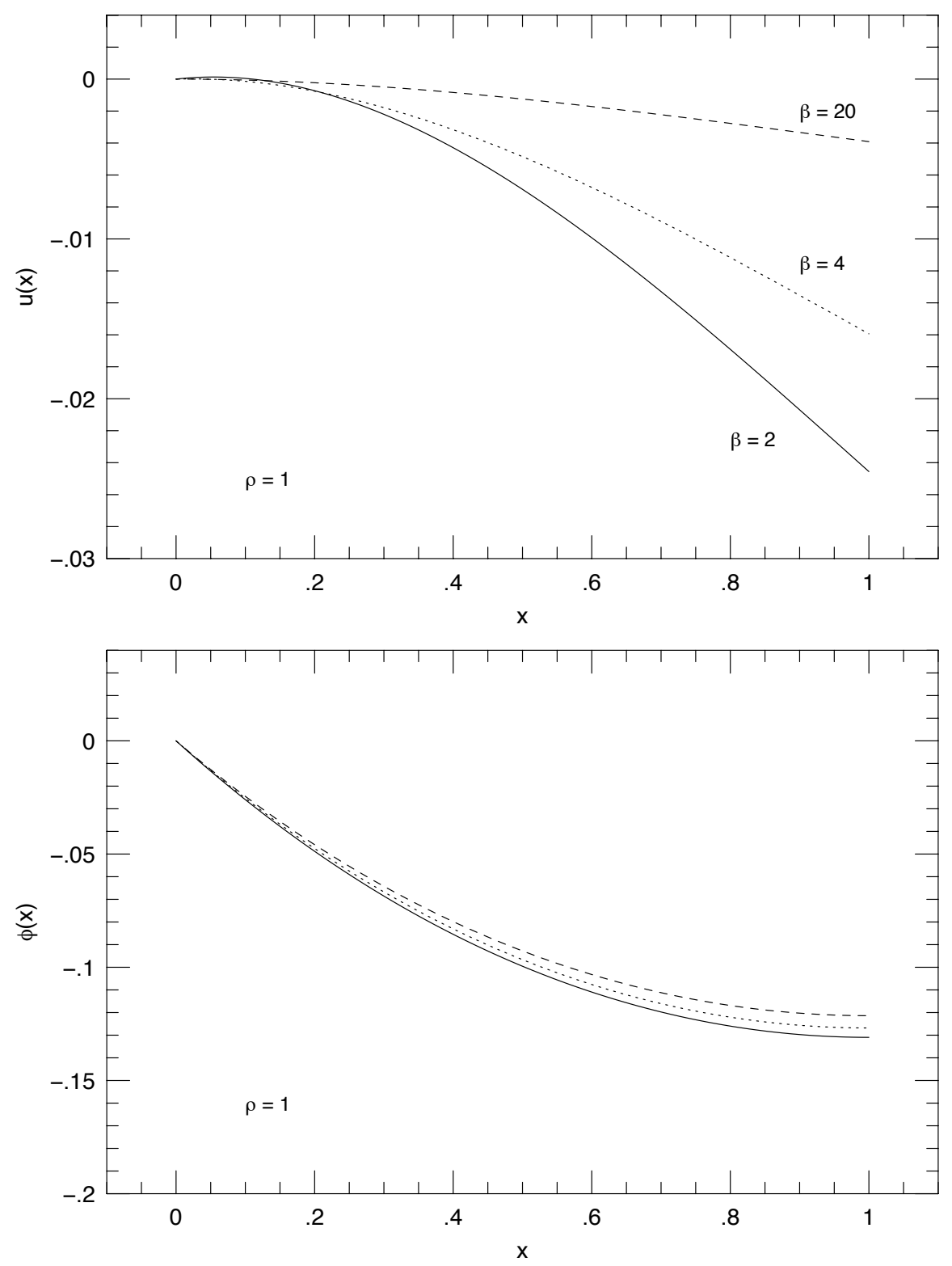

Figure 3. Vertical displacement and angular rotation when $\theta_{A}=-20$ and $\rho=1$. 


\section{References}

[1] J. Ahn, D.E. Stewart - A viscoelastic Timoshenko beam with dynamic frictionless impact, Discrete and Continuous Dynamical Systems Series B 12 (2009), 1 -22.

[2] C. Bernardi, Y. Maday, F. Rapetti - Discrétisations variationnelles de problèmes aux limites elliptiques, Collection "Mathématiques et Applications" 45, Springer-Verlag (2004).

[3] M.I.M. Copetti - A numerical method for a nonlinear system of integro-differential equations arising in thermoviscoelasticity, Comp. Methods Appl. Mech. Engrg. 196 (2007), 1085-1094.

[4] M.I.M. Copetti, J.R. Fernández - Finite element approximation to a contact problem for a nonlinear thermoviscoelastic beam, J. Math Anal. Appl. 383 (2011), 506-521.

[5] L. El Alaoui, A. Ern, M. Vohralík - Guaranteed and robust a posteriori error estimates and balancing discretization and linearization errors for monotone nonlinear problems. Comput. Methods Appl. Mech. Engrg. 200 (2011), 2782-2795.

[6] V. Girault, P.-A. Raviart - Finite Element Methods for Navier-Stokes Equations, Theory and Algorithms, Springer-Verlag (1986).

[7] J.E. Lagnese, G. Leugering, E.J.P.G. Schmidt - Modelling of dynamic networks of thin thermoelastic beams, Math. Meth. in Appl. Sci. 16 (1993), 327-358.

[8] T.F. Ma - Existence results and numerical solutions for a beam equation with nonlinear boundary conditions, Appl. Numer. Math. 47 (2003), 189-196.

[9] J. Peradze - A numerical algorithm for a Kirchhoff-type nonlinear static beam, J. Appl. Math. 2009 (2009), 12pp.

[10] M.H. Sapir, E.L. Reiss - Dynamic buckling of a nonlinear Timoshenko beam, SIAM J. Appl. Math. 37 (1979), 290-301.

[11] R. Verfürth - A Posteriori Error Estimation Techniques for Finite Element Methods, Oxford University Press (2013).

[12] M.C. Zelati, C. Giorgi, V. Pata - Steady states of the hinged extensible beam with external load, Math. Models. Meth. in Appl. Sci. 20 (2010), 43-58. 